\title{
Les financements de la prise en charge du VIH/SIDA dans les pays en développement : le cas de l'Afrique Sub-saharienne
}

\author{
Salmata Ouedraogo, Ph. D. \\ Université du Québec à Chicoutimi \\ Sandrine Aïda Koissy-Kpein, Ph. D. \\ Centre d'études de populations, de pauvreté et de politiques socio-économiques, Luxembourg
}

\section{INTRODUCTION}

\section{Le VIH/SIDA, lequel a son impact dramatique sur le développement humain et économique, constitue un obstacle à la croissance et au développement économique des pays. Les traitements pour ce fléau se prennent toute la durée de la vie, ce qui nécessite un énorme effort financier à court et à long terme.}

Le VIH/SIDA, lequel a son impact dramatique sur le développement humain et économique, constitue un obstacle à la croissance et au développement économique des pays. Les traitements pour ce fléau se prennent toute la durée de la vie, ce qui nécessite un énorme effort financier à court et à long terme. Plus que jamais, la lutte contre cette maladie constitue, aussi bien dans les pays riches que dans les pays en développement (PED), un enjeu majeur de santé publique. C'est ainsi qu'elle figure parmi les Objectifs du Millénaire pour le Développement (OMD) énoncés par les Nations Unies ${ }^{a}$. Elle représente, à bien des égards, l'un des secteurs de santé publique où les avancées ont été les plus spectaculaires au cours des dernières décennies. Par exemple, depuis les années 1980/1990 où l'épidémie a commencé à causer de sérieux dégâts à la fois sur le plan humain, économique, social et politique, des progrès considérables ont été observés dans les pays pauvres en général, et en Afrique Subsaharienne en particulier. Ce succès est dû à de nombreux facteurs, dont la prévention et le traitement de la maladie dans les populations à risques. Un autre facteur déterminant dans la lutte contre le VIH/SIDA réside dans le succès de nombreux programmes d'accès aux traite- ments antirétroviraux (ARV) mis en œuvre dans les années 1990/2000 en Afrique Sub-saharienne, en Amérique Latine/Caraïbes et en Asie. Ces programmes ont été mis en place, soit sous l'initiative du Programme Commun des Nations Unies sur le VIH/SIDA (ONUSIDA), soit dans le cadre d'une initiative gouvernementale ${ }^{1}$.

De même, le lancement de diverses initiatives internationales (le Fonds mondial pour la lutte contre le SIDA, la tuberculose et le paludisme, le Plan d'urgence du Président pour l'Aide dans la lutte contre le VIH/SIDA (PEPFAR), le Programme multi-pays sur le SIDA de la Banque mondiale, l'UNITAID International Drug Purchase Facility ${ }^{\mathrm{b}}$ ) et la production croissante de médicaments génériques à coût réduit ont conduit à une baisse significative du prix de la plupart des schémas thérapeutiques de première intention de $30 \%$ à $64 \%$, de 2004 à 2007, dans les pays à revenu faible ou intermédiaire. Ces initiatives ont permis, par ailleurs, une augmentation significative de la couverture en ARV dans ces différents pays, la proportion de personnes nécessitant la mise sous traitements étant passée de $33 \%$ en 2007 à $42 \%$ en $2008^{2}$.

Toutefois, en dépit de ces progrès, d'importants défis doivent être encore relevés dans les prochaines années pour parvenir à l'accès universel aux traitements ARV. Particulièrement dans les pays d'Afrique Sub-saharienne, où environ 3 millions de patients infectés par le VIH y reçoivent des traitements ARV sur un total de 7 millions en besoin, soit une proportion de $43 \%{ }^{2}$.

En outre, il est important de noter que, dans ce contexte, le passage aux traitements ARV de 
seconde ligne pour un certain nombre de patients (surtout ceux présentant des cas de résistance) s'avère très coûteux ${ }^{3}$. Celui-ci induit une dépense plusieurs fois supérieure au revenu par habitant, lorsque tous les coûts associés aux traitements sont pris en compte. Or, du fait de leur faible niveau de ressources disponibles, nombreux sont les PED qui se trouvent de plus en plus en difficulté pour répondre aux besoins les plus urgents de leurs populations en matière de traitements et de soins contre le VIH/SIDA ${ }^{2}$. C'est pourquoi, en complément de l'aide reçue des bailleurs de fonds internationaux, certains PED tentent de mettre en place des systèmes de financements communautaires impliquant les bénéficiaires. Ces systèmes basés sur le pré- paiement et le partage des risques sont expérimentés depuis un certain nombre d'années dans les pays d'Afrique Sub-saharienne. Par ailleurs, le secteur public de prise en charge des personnes infectées par le VIH et les entreprises privées, dans certains pays, ont mis en place des stratégies de financements fondées sur des partenariats public-privé. Ces deux perspectives, présentées généralement comme des alternatives à la participation financière des ménages pratiquée sur le lieu de délivrance des services de soins, sont très encouragées par les bailleurs de fonds (PEPFAR ${ }^{\mathrm{c}}$, Fonds mondial, Banque mondiale). Mais, sont-elles équitables, efficientes et durables?

\section{INSUFFISANCE D'INFRASTRUCTURES, DE RESSOURCES FINANCIERE ET HUMAINES DANS LES SYSTEMES DE SANTE EN AFRIQUE SUB-SAHARIENNE}

Le rapport de l'ONUSIDA souligne que

l'Afrique Sub-saharienne reste surtout la région la plus durement touchée par le VIH. En 2010, près de $68 \%$ de toutes les personnes vivant avec le VIH résidaient en Afrique Sub-saharienne, une région qui ne représente que $12 \%$ de la population mondiale.

Le SIDA a été qualifié de «crise sans précédent... qui nécessite une riposte sans précédent. » ${ }^{4}$. Selon le rapport de l'ONUSIDA (2010), à l'échelle mondiale, le nombre de personnes vivant avec le VIH/SIDA, en fin 2010, était estimé à 34 millions (hausse de $17 \%$ par rapport à 2001), avec 2,7 millions de nouvelles infections et 2 millions de décès par an. Le pourcentage de femmes parmi les personnes vivant avec le VIH reste stable à $50 \%$, bien qu'elles soient plus touchées que les hommes, notamment en Afrique Sub-saharienne (59\%). Le rapport de l'ONUSIDA souligne que l'Afrique Sub-saharienne reste surtout la région la plus durement touchée par le VIH. En 2010, près de $68 \%$ de toutes les personnes vivant avec le VIH résidaient en Afrique Sub-saharienne, une région qui ne représente que $12 \%$ de la population mondiale. La région était également à l'origine de $70 \%$ des nouvelles infections en 2010, bien qu'on ait enregistré une baisse notable de ce taux dans cette partie du monde.
Depuis 1998, le VIH/SIDA a fauché un million de vies au moins par an en Afrique Sub-saharienne. Néanmoins, les décès diminuent régulièrement depuis cette date car l'accès aux traitements antirétroviraux gratuits s'est généralisé dans la région.

$\mathrm{Au}$ cours de la présente décennie, il a été enregistré une hausse sans précédent de l'accès au traitement contre le VIH dans les pays à ressources limitées, où l'on ne disposait pas de médicaments jusqu'alors ${ }^{2}$. En fin 2011, plus de 8 millions de personnes (dont 562000 enfants) vivant avec le VIH étaient sous thérapie antirétrovirale dans les pays à revenu faible ou intermédiaire. Toutefois, pour atteindre la cible des 15 millions de personnes sous traitement antirétroviral d'ici 2015, l'OMS souligne que plus de 7 millions de personnes doivent être inscrites dans un programme de traitement.

Depuis le début de la maladie, des progrès considérables ont été observés particulièrement en Afrique Sub-saharienne pour pallier les nombreuses conséquences à la fois sur le plan humain, économique, social et politique du fléau. Ce succès est dû à de nombreux facteurs, dont la prévention et le traitement de la maladie dans les populations à risques. Un autre facteur déterminant dans la lutte contre le VIH/SIDA réside dans le succès de nombreux programmes 
d'accès aux traitements antirétroviraux mis en œuvre dans les années 1990/2000 dans les pays à faible revenu, particulièrement en Afrique Subsaharienne. Ces différentes stratégies ont eu comme résultats une baisse du coût des médicaments antirétroviraux et la multiplication des programmes internationaux tels que le Fonds Mondial de lutte contre le SIDA, la tuberculose et le paludisme (GFATM), le Presidents's Emergency Plan for AIDS Relief et l'Initiative internationale d'achats de médicaments (UNITAID).

\section{Un autre facteur déterminant dans la lutte contre le VIH/SIDA réside dans le succès de nombreux programmes d'accès aux traitements antirétroviraux mis en ouvre dans les années 1990/2000 dans les pays à faible revenu, particulièrement en Afrique Sub-saharienne.}

Malgré les efforts considérables engagés par les acteurs nationaux et internationaux de la lutte contre le VIH/SIDA dans les pays à ressources limitées, l'accès à la prise en charge des personnes vivant avec le VIH/SIDA demeure un problème de santé publique majeur ${ }^{5,6}$. Dans la plupart de ces pays, le financement de la prise en charge du VIH/SIDA repose essentiellement sur la contribution financière des ménages ${ }^{7}$. Ces derniers participent, généralement, pour plus de $50 \%$ au financement global de la prise en charge, faisant ainsi face à des dépenses de santé désastreuses ${ }^{8}$.

En Afrique Sub-saharienne, où l'on observe les plus faibles pourcentages de dépenses publiques consacrées à la santé, plus de la moitié des dépenses totales de santé sont prises en charge par les ménages sous la forme de paiements directs. Les ménages sont contraints, par conséquent, de payer directement les soins de leur poche ${ }^{9}$. Ces coûts sont dramatiquement élevés et constituent pour ces ménages, des dépenses de santé perçues comme «catastrophiques » lorsqu'elles se rapportent à la prise en charge de l'infection à VIH/SIDA ${ }^{10,8,11,12}$.

À ces dépenses catastrophiques de santé s'ajoutent les frais funéraires, la garde des Orphelins et Enfants et Vulnérables, la perte de la productivité, etc. Ces ménages sont alors contraints d'utiliser de façon plus «rationnelle» leur revenu de base et de ce fait, ils modifient leurs habitudes de consommation, d'épargne et d'investissement, vendent des actifs de production ou contractent des prêts d'urgence pour faire face aux coûts de la vie $e^{13,14,15}$. Ce comportement plonge ces ménages davantage dans le cercle vicieux de la pauvreté et les rend encore plus vulnérables au VIH/SIDA. Comme le soulignent Beaulière et al. ${ }^{16}$, «les ménages les plus pauvres font face à une barrière financière les empêchant d'accéder aux soins contre le VIH en général, et aux traitements antirétroviraux (ARV) en particulier malgré la distribution de plus en plus gratuite de ces derniers dans la plupart des pays en développement ».

Bien avant l'apparition de l'épidémie de VIH/SIDA, le secteur de la santé publique en Afrique Sub-saharienne souffrait déjà d'une pénurie de professionnels avec des capacités de diagnostic, de prévention et de traitement limitées des nombreuses maladies. En raison des mauvaises conditions de travail et des bas salaires, les taux de rétention des médecins et des infirmières dans le secteur public de nombreux pays d'Afrique Sub-saharienne sont extrêmement bas ${ }^{17,18}$. L'épidémie de VIH/SIDA a non seulement fortement augmenté la demande dans un secteur souffrant déjà de dysfonctionnements, mais a également réduit la main-d'œuvre en raison de la mortalité des professionnels de santé infectés ${ }^{19}$. Selon plusieurs experts, la pénurie du personnel de santé figure parmi les contraintes les plus importantes qui pèsent sur le développement des systèmes de santé dans la région ${ }^{20,18}$. La formation de ces professionnels de la santé pose également problème, puisqu'ils ne sont pas nombreux à posséder l'expertise nécessaire sur le traitement de l'infection par le VIH.

Étant donné que les ressources humaines constituent déjà une véritable contrainte, et que les systèmes sanitaires sont très sollicités en matière de programmes de lutte contre le VIH/SIDA, certains observateurs pensent que les nouveaux mécanismes de financement du VIH/SIDA (tels que le Fonds mondial, le PEPFAR ou l'UNITAID) détournent de façon inefficace les ressources des interventions les plus rentables et pourront même 
causer plus de mal que de bien ${ }^{21}$. Ces financements indiquent qu'un partage plus grand de nouvelles aides internationales doit être dirigé vers des activités de renforcement du système de santét ${ }^{20}$.

Ce contexte montre qu'il est urgent de former des médecins, des infirmières et autres four-

\section{NÉCESSITÉ D’UN AGENDA COMMUN COORDINATION DES FINANCEMENTS}

Il a fallu beaucoup de temps pour parvenir à l'actuel niveau d'engagement financier de la part des pays développés dans la lutte contre le VIH/SIDA. L'épidémie de VIH/SIDA représente un défi d'une immense complexité et nécessite une réponse exceptionnelle. Rischard ${ }^{23}$ souligne que l'épidémie est l'un des problèmes mondiaux qui échappent à notre contrôle de manière exponentielle, alors que le mode de fonctionnement des institutions suit une progression linéaire dans le temps.

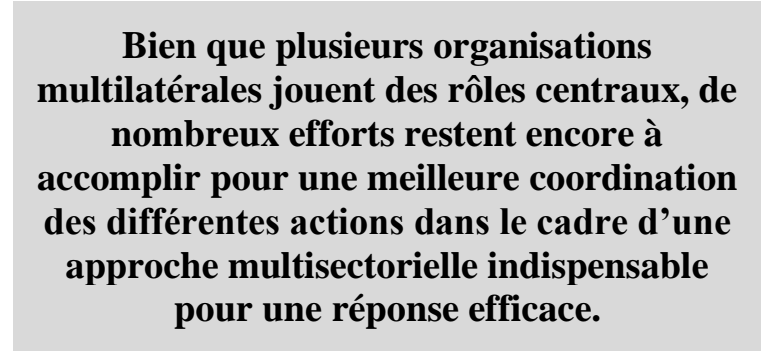

La problématique actuelle est le déficit de leadership et, jusqu'à nos jours, il n'existe pas d'organisation, ni d'institution ou de programme adapté pour combler cette lacune. Bien que plusieurs organisations multilatérales jouent des rôles centraux, de nombreux efforts restent encore à accomplir pour une meilleure coordination des différentes actions dans le cadre d'une approche multisectorielle indispensable pour une réponse efficace. Même si le Fonds Mondial de Lutte contre le SIDA, la Tuberculose et la Malaria (FMSTM) peut se vanter de succès considérables $^{24}$, il reste malheureusement menacé d'insécurité faute de perspectives de financement suffisantes et pérennes à long terme. De plus, malgré la volonté d'implication de multiples parties prenantes et les mécanismes d'offre, et surtout l'effort établis afin d'éviter la prédominance de l'administration gouvernementale, ce fonds ne semble pas affecter assez de nisseurs de soins de santé ${ }^{18}$. Parallèlement, comme le soulignent Grépin et Savedoff ${ }^{22}$, les pays développés devraient cesser de recruter des professionnels de santé dans les pays où le VIH/SIDA représente un fardeau particulièrement lourd de manière à limiter la fuite des cerveaux.

\section{ET D'UN LEADERSHIP DANS LA}

ressources au secteur privé. Ce qui pourrait pourtant jouer un rôle crucial notamment en matière de partenariat public-privé (PPP) dans l'extension de l'accès à la thérapie antirétrovirale et la prise en charge des ayant-droits des travailleurs dans le cadre d'une approche communautaire en Afrique Sub-saharienne ${ }^{25,26,21}$. Selon d'autres analyses, le Fonds Mondial de lutte contre le SIDA, la Tuberculose et la Malaria fonctionne uniquement comme un mécanisme de financement, et ne dispose d'aucune capacité d'exécution et d'assistance technique ce qui constitue une limite supplémentaire importante.

Le PEPFAR, qui représente l'engagement financier le plus important jamais pris par un seul pays pour une initiative sanitaire internationale, adopte une approche différente ${ }^{3}$. En effet, c'est un plan mis en place mettant en relation les organisations et les institutions pouvant fournir une assistance technique d'une part, et entre un certain nombre de gouvernements des pays en développement, d'organisations et d'institutions, d'autre part ${ }^{19}$. Pendant la courte période où le PEPFAR a été opérationnel, environ 400000 personnes ont pu bénéficier d'une thérapie antirétrovirale ${ }^{27}$.

Un point important est à souligner puisqu'à la faveur de la lutte contre le VIH/SIDA, un consensus tend à s'établir, au plan international, pour souhaiter une réduction progressive de la part $\mathrm{du}$ financement reposant sur le paiement direct et une promotion des mécanismes de mutualisation des risques et d'assurance, incluant les systèmes d'assurance maladie universelle ${ }^{28,29,30}$. Dans cette optique, il serait pertinent de mettre en place un mécanisme de financement équitable, efficient et durable des soins et traitements contre le VIH/SIDA, dont la gratuité serait l'axe principal $^{31,8,32}$. 


\section{LES OBSTACLES À SURMONTER DANS LA LUTTE CONTRE LE SIDA}

Si pour éradiquer la variole il a été nécessaire de consentir un effort mondial coordonné avec un partage clair des tâches et des responsabilités, comment peut-on imaginer faire moins que cela pour s'attaquer à cet enjeu autrement plus complexe visant la maîtrise du VIH/SIDA ? ${ }^{33}$.

Pour des actions efficaces, il est urgent de former massivement les professionnels de santé afin de les rendre plus opérationnels dans la lutte contre le VIH/SIDA. Il est aussi nécessaire d'explorer de nouveaux mécanismes de financement à long terme. Mais aussi, faut-il trouver des mécanismes incitatifs pour maintenir et retenir les professionnels de santé qui ont été formés ${ }^{34}$. Ces mécanismes incitatifs peuvent être de nature financière ou non financière. Cela aura comme conséquence importante une meilleure valorisation des professions de santé, une rémunération adéquate et de meilleures perspectives de carrière.

Un autre point important à noter est l'existence d'une nécessité d'utiliser de manière plus efficace les compétences et les potentialités disponibles dans le secteur privé de la santé, mais également la nécessité de mettre en place des programmes pour permettre à ce secteur de bénéficier des financements des donateurs.

Suite à ces remarques, il est crucial de mettre en place des stratégies qui permettront d'élaborer et de coordonner des mécanismes de financement des soins de santé durables pour la majorité de la population, à travers une utilisation efficiente et optimale des ressources locales et exogènes provenant de l'aide au développement.

En Afrique Sub-saharienne, le quintile le plus riche de la population profite bien plus des services publics que le quintile le plus pauvre ${ }^{35}$, alors que l'argent des premiers pourrait contribuer à la mise en place de solides systèmes d'assurance médicale. Pourquoi ne pas utiliser une partie des fonds du Fonds Mondial de lutte contre le SIDA, la Tuberculose et la Malaria ou d'autres fonds des donateurs pour lancer le démarrage de ces systèmes? Cette approche dite verticale d'extension de la thérapie antirétrovirale nécessite des dépenses colossales. Par conséquent, l'adoption d'une approche horizontale pourrait permettre un élargissement de l'accès aux antirétroviraux et fournir ainsi une occasion unique de soutenir les pauvres et de construire des systèmes de santé durables dans les pays à faibles revenus.

La plupart des pays ont du mal à atteindre les cibles du sixième objectif des OMD, qui prévoyaient un accès universel au traitement du VIH/SIDA en 2010, et aussi d'avoir arrêté et inversé la progression du VIH/SIDA d'ici 2015. Le rapport, publié avant la tenue de la XIX $^{\mathrm{e}}$ conférence internationale sur le SIDA, présente les chiffres actuels du VIH/SIDA. Il est estimé dans le rapport que 34,2 millions de personnes vivent avec le SIDA en 2011. En 2010, l'ONUSIDA a indiqué qu'au moins 56 pays étaient parvenus à stabiliser ou à réduire de manière considérable le taux des nouvelles infections par le VIH. En effet, cette tendance s'est confirmée puisque le nombre des nouvelles infections par le VIH a baissé de près de $20 \%$ au niveau mondial au cours des 10 dernières années.

On estime que le nombre de personnes nouvellement contaminées est passé de 3 millions en 2001 à 2,9 millions en 2010. Le nombre d'individus infectés devrait continuer à augmenter lentement en Afrique Subsaharienne et rester à peu près au niveau actuel au plan mondial, du fait des thérapies antirétrovirales, qui prolongent la vie des patients, et d'une croissance soutenue de la population.

Il est plus qu'urgent de mettre en œuvre un programme de prévention efficace pour inverser la progression du SIDA ${ }^{3}$. En 2008, les Nations Unies soulignaient que le rapport coût-efficacité de la prévention est 28 fois supérieur à celui associé au traitement et qu'un ensemble de mesures de prévention du VIH permettrait d'éviter plus de $63 \%$ de nouvelles infections prévues les dernières années. 


\section{ESTIMATION DES BESOINS DE FINANCEMENT DE LA LUTTE CONTRE LE VIH/SIDA}

\author{
Le VIH/SIDA entretient également une \\ épidémie de tuberculose, maladie \\ infectieuse la plus meurtrière pour les \\ personnes vivant avec le VIH (responsable \\ de $13 \%$ des décès dus au SIDA à travers le \\ monde selon l'OMS). Les deux maladies \\ sont si étroitement liées que l'on parle très \\ souvent de co-épidémies qui s'entraînent et \\ se renforcent mutuellement.
}

Selon les estimations, le financement requis pour 2007 varie entre 10 et 20 milliards de dollars US $^{36}$. De l'avis de nombreux observateurs, ces projections «moyennes » sont trop conservatrices. Les projections portaient sur les besoins éventuels dans les domaines de la prévention, du traitement, des soins et du soutien ainsi que l'élaboration des politiques, la défense des intérêts, la recherche opérationnelle, l'administration, le suivi et l'évaluation. Selon le CISD, les prévisions ne couvraient pas la majeure partie de la recherche fondamentale et clinique liée au VIH/SIDA, ni les travaux sur les nouvelles techniques de prévention telles que les vaccins ou les microbicides. De plus, elles ne tenaient compte ni des coûts de renforcement des infrastructures nationales, ni la mise en place de systèmes sanitaires équipés, fonctionnels avec du personnel compétent et motivé garantissant une couverture universelle aux populations pauvres.

En effet, l'épidémie du VIH/SIDA affecte pratiquement toutes les populations et tous les pays. Mais en général, ce sont les systèmes de santé qui subissent les effets les plus dramatiques. Selon le Population Reference Bureau, l'épidémie a paralysé les systèmes de santé déjà faibles en Afrique, surtout en Afrique Sub-saharienne. Les coûts des traitements de la maladie et des infections «opportunistes » continuent à augmenter. L'affectation de ressources déjà limitées au
VIH/SIDA détourne l'attention d'autres préoccupations de santé, et avec la raréfaction des fonds publics pour les dépenses de santé, les coûts sont transférés en proportion sans cesse croissante au secteur privé, aux ménages et aux particuliers. L'épidémie a, par conséquent, contribué à une érosion progressive du secteur de la santé publique dans ces pays. Le VIH/SIDA entretient également une épidémie de tuberculose, maladie infectieuse la plus meurtrière pour les personnes vivant avec le VIH (responsable de $13 \%$ des décès dus au SIDA à travers le monde selon l'OMS). Les deux maladies sont si étroitement liées que l'on parle très souvent de co-épidémies qui s'entraînent et se renforcent mutuellement. Le VIH active la tuberculose latente chez l'individu, qui devient ensuite infectieux et capable de contaminer d'autres personnes. Cette co-infection contribue à une hausse importante des dépenses de santé et des ressources nécessaires pour lutter contre la maladie.

Il faut souligner qu'en dépit des progrès accomplis ces dernières années afin de combler le « déficit de financement », la mise en place de programmes efficaces a fait défaut. La lutte contre le VIH/SIDA nécessite une appropriation générale et un plan d'action basé sur une approche pragmatique et globale $^{21}$.

L'enjeu est vraiment considérable, mais le passage progressif à l'échelle de la couverture en antirétroviraux fournit une occasion unique de soutenir les pauvres et de construire des systèmes de santé durables dans les PED en général et en Afrique Sub-saharienne en particulier ${ }^{20,21}$. Pour atteindre ces objectifs, les Nations Unies ont estimé qu'il fallait 18,1 milliards de dollars US pour 2007 et 22 milliards de dollars US pour $2012^{\mathrm{d}}$. Pour la première fois, ces projections comprennent certains coûts destinés à renforcer les infrastructures dans les pays touchés.

\section{TENDANCES NOUVELLES DANS LA LUTTE CONTRE LE VIH/SIDA}

La lutte contre le SIDA a suscité ces dernières années une mobilisation sans précédent de la communauté internationale, marquée notamment par la tenue en juin 2001 d'une session extraordinaire de l'Assemblée générale des
Nations Unies ${ }^{\mathrm{e}}$, le lancement en 2002 du Fonds mondial de lutte contre le SIDA, la tuberculose et le paludisme et en 2003 de l'initiative « Three by Five » par l'OMS et l'ONUSIDA. Cette mobilisation s'est aussi accompagnée d'une 
forte progression des ressources globales consacrées à la lutte contre la pandémie de VIH, réparties à peu près à moitié entre l'aide extérieure et les ressources propres des pays bénéficiaires.

Comparativement aux pays développés où les avantages du traitement antirétroviral hautement actif (HAART) sont reconnus depuis plusieurs années, des engagements politiques ont été pris afin de rendre disponible l'HAART également dans les pays à faible niveau de revenu. Ces engagements visent à inclure le traitement antirétroviral comme une composante essentielle de la lutte contre le VIH/SIDA. Dans cette optique, une importante baisse de prix des antirétroviraux pour les pays les plus pauvres a été obtenue grâce à diverses initiatives internationales (Fonds mondial, PEPFAR, Programme multi-pays sur le SIDA de la Banque mondiale, UNITAID International Drug Purchase Facility) et la production croissante de médicaments génériques à coût réduit ${ }^{37}$. La baisse significative du prix de la plupart des schémas thérapeutiques de première intention a été de $30 \%$ à $64 \%$, de 2004 à 2007, dans les pays à revenu faible ou intermédiaire. Ainsi, l'Organisation Mondiale de la Santé (OMS) a intégré les antirétroviraux dans la liste des médicaments essentiels et formulé les directives pour le développement d'une approche de santé publique pour le traitement des infections par le VIH dans les pays en développement ${ }^{38}$.

Soulignons que l'aide extérieure accordée aux pays les plus touchés par le VIH/SIDA ne couvre pas la totalité de leurs besoins. Il faudra des ressources supplémentaires pour financer les coûts récurrents des programmes de lutte contre le VIH/SIDA dans les pays les plus pauvres. Par conséquent, un accroissement significatif de l'aide publique au développement (APD) est nécessaire pour soutenir la lutte contre la pandémie et réaliser les OMD. Pour l'UNITAID, il faut des mécanismes innovants, c'est-à-dire, davantage de ressources, mais également un nouveau type de ressources, plus stables et plus prévisibles, plus pérennes afin de financer les coûts récurrents des programmes de développement humain des pays en développement. Ces mécanismes innovants pourraient être soit un prélèvement à chaque utilisation de moyens de paiement ou un versement par les banques d'un pourcentage des sommes perçues lors de l'utilisation par leurs clients des moyens de paiement qu'elles proposent. Mais cette idée basée sur la taxation des transactions financières ne recueille pas le consentement de tous les pays ${ }^{2}$.

Le rapport publié par l'ONUSIDA ${ }^{39}$ intitulé "What countries need: Investments needed for 2010 targets », prévoit qu'environ un tiers de l'investissement de 25 milliards de dollars US sera d'origine nationale. Les investissements extérieurs bilatéral et multilatéral devront couvrir les 17 milliards restants. Plus de 9 milliards seront alloués au renforcement de systèmes de santé, et 9 autres milliards serviront à fournir des services de santé dédiés au VIH. Le rapport stipule que ces montants permettront :

- d'éviter 2,6 millions de nouvelles infections par le VIH, réduisant ainsi l'incidence du VIH d'environ $50 \%$;

- d'empêcher 1,3 million de décès dans les deux prochaines années;

- d'offrir un traitement antirétroviral à 6,7 millions de personnes;

- d'offrir un dépistage et un service de prévention de la transmission mère-enfant (PTME) à 70 millions de femmes enceintes.

- de réaliser des programmes de prévention du VIH qui toucheront 20 millions d'hommes ayant des rapports sexuels avec des hommes, 7 millions de professionnel(le)s du sexe et 10 millions de consommateurs de drogues injectables;

- de distribuer 8,1 milliards de préservatifs masculins et féminins;

- d'offrir des programmes d'appui social à 7 millions d'orphelins et d'enfants vulnérables.

En ce qui concerne la prise en charge du VIH/SIDA dans les pays en développement, diverses sources permettent le financement. On peut citer, en l'occurrence, l'aide publique internationale au développement, l'aide privée et la contribution des ménages sans oublier l'apport des gouvernements à travers les budgets de l'État ${ }^{40}$. En effet, en Afrique Sub-saharienne, environ $60 \%$ du financement des soins de santé provient de sources privées, et environ $50 \%$ des dépenses totales de santé vont à des prestataires 
privés, souvent sous la forme de paiements directs (out-of-pocket-payments) des pauvres ${ }^{41}$. Depuis les dernières décennies, les politiques de financement de la santé dans les PED ont été surtout marquées par trois principales orientations à savoir la gratuité des soins, la participation financière des usagers et le partage des risques dans des mécanismes assuranciels. Nous allons nous attarder sur cette dernière orientation qui a montré ses preuves dans certains PED. Ces stratégies de financement de la prise en charge du VIH/SIDA dans les pays en développement fondées sur les mécanismes assuranciels sont variées telles qu'illustrées à la figure 1.

Depuis les dernières décennies, les politiques de financement de la santé dans les PED, ont été surtout marquées par trois principales orientations à savoir la gratuité des soins, la participation financière des usagers et le partage des risques dans des mécanismes assuranciels.

Figure 1 - Financement de la prise en charge du VIH/SIDA

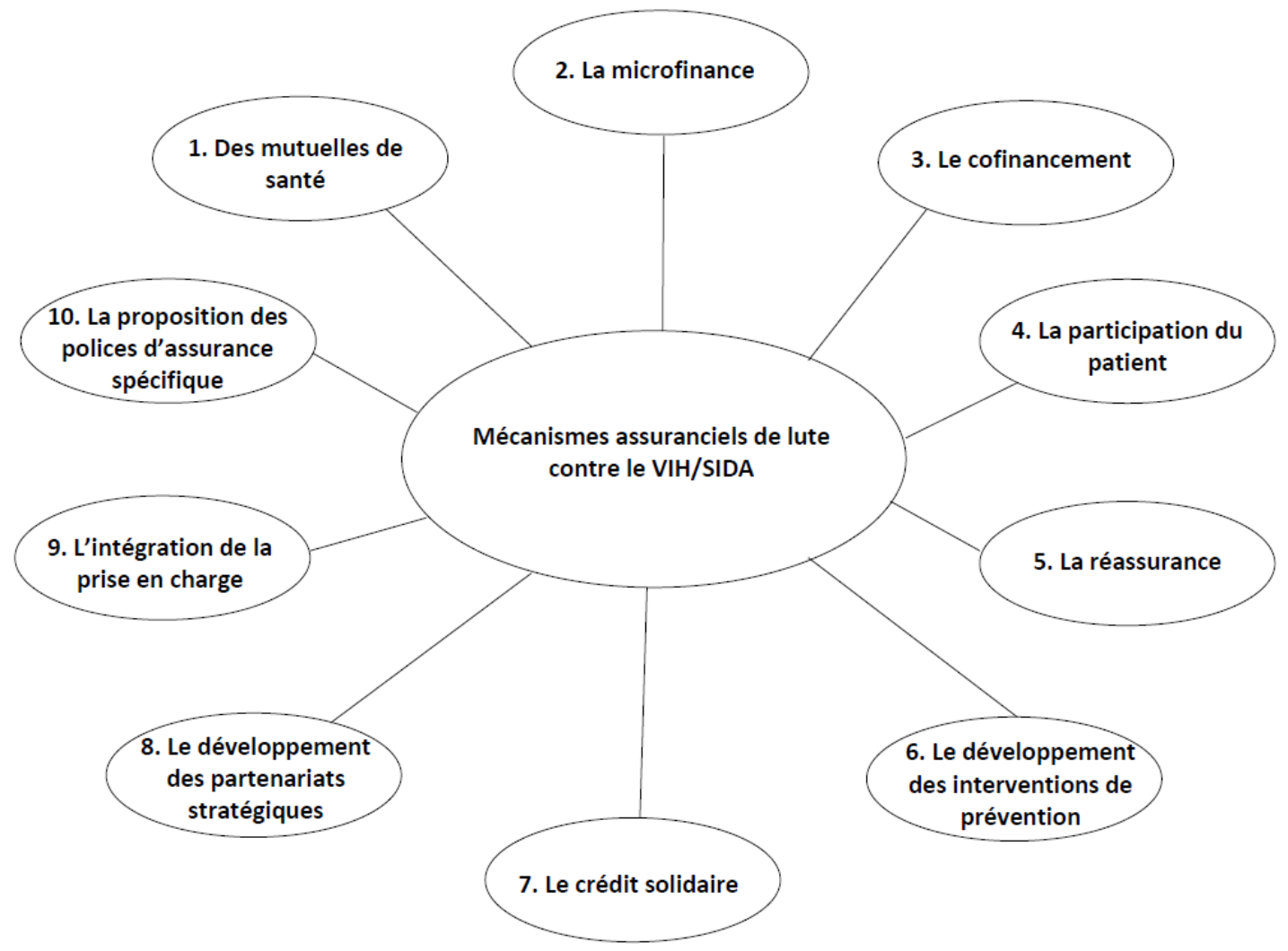

\section{Des mutuelles de santé}

Dans la plupart des pays d'Afrique de l'Ouest, des mutuelles de santé à base communautaire ont été créées. Elles mettent en place une gouvernance fondée sur la représentation des adhérents, elles passent des conventions avec les formations publiques, privées et communautaires de soins, et elles privilégient des garanties portant sur la couverture du petit risque, de la grossesse et de l'accouchement. Ce mécanisme a fait ses preuves surtout au Ghana et au Rwanda. Il peut demander des subsides ou des ressources additionnelles qui proviennent de l'État, à travers les taxes ou l'appui budgétaire des pays donateurs, des institutions internationales, des fonds internationaux (le Global Fund, Bill Gates Foundation, ou le Global Social Trust au Ghana). 


\section{La microfinance}

Initialement conçue pour la lutte contre la pauvreté et le renforcement du pouvoir économique des ménages, les structures de microfinance interviennent désormais en matière de prévention et de prise en charge du VIH/SIDA notamment auprès de leurs clients.

Depuis quelque temps, les initiateurs de la microfinance s'intéresse à la pertinence et à la faisabilité de la prise en compte du VIH/SIDA au nombre des produits offerts aux populations. Les analystes sont alors parvenus à la conclusion que non seulement la microfinance pourrait contribuer à préparer les ménages dans le cas où ils seraient confrontés à l'infection par le VIH, mais également contribuer à la diminution de son impact sur eux. Ainsi, initialement conçue pour la lutte contre la pauvreté et le renforcement du pouvoir économique des ménages, les structures de microfinance interviennent désormais en matière de prévention et de prise en charge du VIH/SIDA notamment auprès de leurs clients. À cet effet, elles développent des produits spécifiques (crédits, épargnes et assurances) et des services de prévention et de prise en charge en collaboration avec les organismes spécialisés dans le domaine. De nos jours, les produits de la microfinance (microcrédits, prêts spécifiques, etc.) sont largement utilisés par l'assurance-santé pour financer la prise en charge du VIH/SIIDA ${ }^{42}$. Il a été également indiqué que les services de microfinance ralentissent la propagation $\mathrm{du}$ VIH/SIDA par le renforcement économique des pauvres, en particulier les femmes ${ }^{43}$.

\section{Le cofinancement}

C'est une stratégie utilisée actuellement par les compagnies d'assurance-santé pour financer la prise en charge du VIH/SIDA. D'une manière générale, la gratuité des ARV tend à être généralisée dans les pays en développement ainsi que d'autres soins qui sont pris en charge gratuitement par les partenaires, les programmes ou par l'État. Lorsqu'elles intègrent les services liés au VIH/SIDA dans leurs polices d'assurance, les compagnies les comptabilisent séparément pour se faire rembourser par des mécanismes externes dont l'État, les partenaires au développement, les programmes verticaux, les $\mathrm{ONG}^{45,44}$.

\section{La participation du patient}

La participation du patient aux dépenses de santé (le co-paiement) pour minimiser l'usage excessif des services par les malades ${ }^{44}$.

\section{La réassurance}

La réassurance ou les crédits octroyés par les institutions de microfinance aux personnes vivant avec le VIH/SIDA. C'est l'une des stratégies utilisées par les compagnies d'assurance-santé ou de microfinance pour financer la prise en charge du VIH/SIDA dans les pays en développement.

\section{Le développement des interventions de prévention}

Le développement des interventions de prévention du VIH/SIDA qui est utilisé très souvent comme stratégie par certaines compagnies d'assurance-santé pour vulgariser l'accès aux services de VIH/SIDA. Ce sont notamment la sensibilisation et le dépistage du VIH pouvant conduire aux traitements antirétroviraux.

\section{Le crédit solidaire}

Le crédit solidaire qui permet aux personnes vivant avec le VIH/SIDA d'avoir accès au crédit tant qu'elles ont la force physique pour contracter un prêt.

\section{Le développement des partenariats stratégiques}

Le développement des partenariats stratégiques avec les institutions spécialisées dans la lutte contre le VIH/SIDA pour offrir des services d'information, de counseling et de dépistage du $\mathrm{VIH}$, de soins et traitement en lien avec le SIDA ${ }^{44}$.

\section{L'intégration de la prise en charge}

L'intégration de la prise en charge du VIH/SIDA dans l'assurance commerciale qui est également une stratégie utilisée par les compagnies.

\section{La proposition des polices d'assurance spécifique}

Enfin, la proposition des polices d'assurance spécifique pour le VIH/SIDA. La plupart des assureurs proposent maintenant des polices d'assurance spécifiques avec le prix correspondant pour la couverture des services liés au VIH/SIDA et même quelquefois, des prêts relatifs aux cérémonies funèbres. 


\section{CONCLUSION}

Il est important de rappeler que l'accès abordable à des soins de santé de qualité est un droit fondamental pour tous les individus. C'est un droit essentiel qui est favorable à la croissance économique, à l'éradication de la pauvreté et au développement des pays. De nos jours, d'énormes efforts sont consentis aussi bien par les acteurs nationaux qu'internationaux dans le but d'améliorer les indicateurs de santé des pays dans l'optique de l'atteinte des OMD. Malgré ces efforts, d'énormes difficultés subsistent dans la recherche de solutions pour répondre aux besoins des populations en matière de soins de santé dans la lutte contre le VIH/SIDA, particulièrement dans les pays à faible et moyen revenu. Les différentes analyses montrent la nécessité d'investir davantage dans de nouvelles ressources économiques, financières et humaines pour résoudre efficacement et globalement le problème du VIH/SIDA. En plus d'injecter de nouveaux fonds à long terme dans les programmes de lutte contre le $\mathrm{VIH} / \mathrm{SIDA}$, tous les acteurs nationaux comme internationaux doivent respecter leurs engagements. À titre d'exemple, les bailleurs de fonds internationaux devraient établir un calendrier clair et précis indiquant comment ils vont atteindre l'objectif visant à consacrer $0,7 \%$ $\mathrm{du}$ revenu national brut à l'aide publique au développement. De plus, les acteurs nationaux devraient avoir une volonté politique pour la lutte contre le fléau et une bonne gestion des fonds destinés à son éradication, puisque le VIH/SIDA a des conséquences macroéconomiques, humaines et politiques catastrophiques.

\section{BIBLIOGRAPHIE}

${ }^{1}$ Katzenstein, D., Laga, M. and Moatti, J.P. (2003). "The Evaluation of the HIV/AIDS Drug Access Initiatives in Cote d'Ivoire, Senegal and Uganda: How Access to Antiretroviral Treatment can Become Feasible in Africa." AIDS, 17 Suppl 3, S1-4.

${ }^{2}$ OMS, ONUSIDA, UNICEF (2009). «Vers un accès universel: étendre les interventions prioritaires liées au VIH/SIDA dans le secteur de la Santé : rapport 2009. » Genève : OMS

${ }^{3}$ Over, M. (2009). «Échec de la prévention : le poids croissant des droits engendrés par l'aide américaine au financement du traitement mondial contre le VIH/SIDA et la voie à suivre. » Revue d'économie du développement, 23 : 107-144.

${ }^{4}$ Piot, P. (2003). "The Need for an Exceptional Response to an Unprecedented Crisis. ". Presidentials Fellows Lecture. Washington DC: World Bank (consulté le 16/04/2010) URL:,http://data.unaids.org/ Media/Speeches02/piot_worldbank_20nov03_en.pdf

${ }^{5}$ Nemes, M.I., Beaudoin, J., Conway, S., Kivumbi, G.W., Skjelmerud, A., Vogel, U. (2006). "Evaluation of Who's Contribution to "3 by 5": Main Report. Geneva: World Health Organization 2006.

${ }^{6}$ Posse, M., Meheus, F., Asten, H.V., van der Ven, A., Baltussen, R. (2008). "Barriers to Access to Antiretroviral Treatment in Developing Countries: a Review." Trop Med Int Health 13(7): 904-913.

${ }^{7}$ Zachariah, R., Van Engelgem, I., Massaquoi, M., Kocholla, L., Manzi, M., Suleh, A., Phillips, M. and Borgdorff, M. (2008). "Payment for Antiretroviral Drugs is Associated with a Higher Rate of Patients Lost to Follow-up Than Those Offered Free-of-Charge Therapy in Nairobi, Kenya." Trans R Soc Trop Med Hyg 102(3): 288293.

${ }^{8}$ Souteyrand, Y.P., Collard, V., Moatti, J.P., Grubb, I., Guerma, T., (2008). Free Care at the Point of Service Delivery. A Key Component for Reaching Universal Access to HIV/AIDS Treatment in Developing Countries. AIDS; 22 (Suppl1): S161-168.

${ }^{9}$ Fonteneau, B. (2005). Faire face au VIH/SIDA. Contribution potentielle des institutions de microfinance et des systèmes de microassurance santé. Université Catholique de Louvain;VLIR/DEGOS. (Consulté le 27/08/2010) URL : http://www.sensoa.be/pdf/internationaal_parlementair/faire_face_au_vih_sida.pdf.

${ }^{10}$ Beaulière, A., Touré, S., Koné, K., Pouhé, A., Kouadio, B., N'Dri-Yoman, T., Dabis, F., Traoré-Ettiegne, Eholié, S. and Anglaret, X. (2009). "The Financial Burden of Morbidity in HIV-infected Adults on Antiretroviral Therapy in Côte d'Ivoire." Presented at the 5th International AIDS Society (IAS) Conference on HIV Pathogenesis, Treatment and Prevention, Cape Town, South Africa, 19-22 July 2009.

${ }^{11}$ Onwujekwe, O., Onoka, C., Uzochukwu, B., et al. 2009.

Is Community-based Health Insurance an Equitable Strategy for Paying for Healthcare? Experiences from Southeast Nigeria. Health Policy, 92: 96-102.

${ }^{12}$ Boyer, S., Marcellin, F., Ongolo-Zogo, P., Abega, S.C., Nantchouang, R., Spire, B. and Moatti, J.P. (2009). "Financial Barriers to HIV Treatment in Yaounde, Cameroon: First Results of a National Cross-sectional Survey. "Bull World Health Organ.; 87(4):279-87.

${ }^{13}$ Russell, S. (2004). "The Economic Burden of Illness for Households in Developing Countries: a Review of Studies Focusing on Malaria, Tuberculosis, and Human 
Immunodeficiency Virus/Acquired Immunodeficiency Syndrome." Am J Trop Med Hyg 71(2 Suppl): 147-155.

${ }^{14}$ McIntyre, D., Thiede, M., Dahlgren, G., Whitehead, M. (2006). "What Are the Economic Consequences for Households of Illness and of Paying for Health Care in Low- and Middle-Income Country Contexts?" Soc Sc Med; 62:858-65.

${ }^{15}$ Chandaani, T. (2008). Microinsurance for Markets Affected by HIV/AIDS. In: Microfinance and HIV/AIDS Note \#3. USAID. [Consulté le 16/04/2010] URL: < http://www.microlinks.org/ ev_en.php?ID=23473_201\&ID2=DO_TOPIC >.

${ }^{16}$ Beaulière, A., Pouhé, A., Koné, K., Touré, S. and Alexandre, P.K. (2007). "Accès aux soins et aux traitements contre le VIH/SIDA et dépenses de santé catastrophiques des ménages dans les pays en développement: Le cas de la Côte d'Ivoire." 31èmes Journées des Économistes de la Santé Français. 3-4 décembre 2009, Rennes.

[Consulté le 20/06/2012] URL: < http://www.cesasso.org/docs/TextesJESF2009/BEAULIERE.pdf>

${ }^{17}$ PNUD (2004). Rapport sur le développement dans le monde 2004: Cultural Liberty in Today's Diverse World. New York, NY: PNUD.

${ }^{18}$ OMS (2006). Rapport sur la santé dans le monde. Travailler ensemble pour la santé. Genève : Organisation mondiale de la santé (OMS).

${ }^{19}$ Lange, J.M., (2007). Étendre l'accès à la prévention et au traitement du VIH dans un contexte de rareté des ressources : défis et perspectives. Revue d'économie du développement 20 (4): 119-139.

${ }^{20}$ Yu, D., Souteyrand, Y., Banda, M.A., Kaufman, J., Perriëns, J.H., (2008). Investment in HIV/AIDS Programs: Does it Help Strengthen Health Systems in Developing Countries? Global Health, 4(8):1-10.

${ }^{21}$ Schneider, K., Garrett, L.A. (2009). The Evolution and the Future of Donor Assistance for HIV/AIDS. Council on Foreign Relations. AIDS2031 Working papers $\mathrm{N}^{\circ} 19$. Available at: http://www.aids2031.org/pdfs/the $\% 20$ evolution $\% 20$ and $\% 20$ future $\% 20$ of $\% 20$ donor $\% 20$ assistanc e\%20for\%20hiv\%20-\%20aids_19.pdf; accessed April 24, 2012.

${ }^{22}$ Grépin, K.A., Savedoff, W.D., (2009). 10 Best Resources... on Health Workers in Developing Countries. Health Policy and Planning 24: 479-482.

${ }^{23}$ Rischard, J. F., (2002). High Noon: 20 Global Issues, 20 Years to Solve Them. Oxford: The Perseus Press.

${ }^{24}$ Severe, P., Leger, P., Charles, M., Noel, F., Bonhomme, G., Bois, G,. George, E., Kenel-Pierre, S., Wright, P., Gulick, R., Johnson, W.D., Pape, J.W., Fitzgerald, D.W., (2005). Antiretroviral Therapy in a 1000 Patients with AIDS in Haiti. N Engl J Med 2005;353:2325-2334.

${ }^{25}$ FMSTM (Fonds Mondial de lutte contre le SIDA, la Tuberculose et la Malaria). (2005). www.theglobalfund.org.

${ }^{26}$ Rosen, S., Feeley, F., Connelly, P., Simon, J., (2007).

The Private Sector and HIV/AIDS in Africa: Taking Stock of Six Years Applied Research. AIDS; 21 (Suppl 3):S41S51.

${ }^{27}$ OMS (2005). Document de travail de l'OMS, Participation financière des patients sur le lieu de prestation des traitements et des soins contre le VIH/SIDA. Genève : OMS.

${ }^{28}$ Carrin, G., Waelkens, M.P., Criel, B., (2005). Community-Based Health Insurance in Developing Countries: a Study of its Contribution to the Perfor-mance of Health Financing Systems. Trop Med Int Health; 10 (8):799-811.

${ }^{29}$ Carrin, G., James, C., (2006). L'Assurance maladie en Afrique francophone : Améliorer l'accès aux soins et lutter contre la pauvreté. In: Dussault G, Fournier P, Letourmy A, eds. Washingtion, DC, The World Bank, 2006:455-499. ${ }^{30}$ Moatti, J.P. (2008). «Économie de la santé dans les pays en developpement : ce que la lutte contre le sida a changé. » C R Biol.; 331(12):964-72.

${ }^{31}$ McIntyre, D., Thiede, M., Dahlgren, G., Whitehead, M. (2006). "What Are the Economic Consequences for Households of Illness and of Paying for Health Care in Low- and Middle-Income Country Contexts?" Soc Sc Med; 62:858-65.

${ }^{32}$ Beaulière, A., Pouhé, A., Koné, K., Touré, S. and Alexandre, P.K. (2007). "Accès aux soins et aux traitements contre le VIH/SIDA et dépenses de santé catastrophiques des ménages dans les pays en développement: Le cas de la Côte d'Ivoire. » 31èmes Journées des Économistes de la Santé Français. 3-4 décembre 2009, Rennes.

${ }^{33}$ Fenner, F., Henderson, D. A., Arita, I., Jezek, Z., Ladnyi, I. D., (1988). Smallpox and Its Eradication. Genève: Organisation Mondiale de la Santé.

${ }^{34}$ Grepin, Karen, A. and William D. Savedoff. (2009). "10 Best Resources on... Health Workers in Developing Countries." Health Policy and Planning". 24(5):1-4.

${ }^{35}$ Preker, A. S., et Langenbrunner, J. C., (2005). Spending Wisely, Buying Health Services for the Poor. Washington, DC: Banque Mondiale.

${ }^{36}$ Coalition interagence SIDA et développement (2005). Ressources requises pour lutter contre le VIH/SIDA. Site Web: www.icad-cisd.com.

${ }^{37}$ Ventelou, B., Moatti, J.P., (2008). Better is Bigger: Scaling-Up Antiretroviral Policies in Sub-Saharan Africa. Future HIV Therapy, 2 (4), p. 297-299.

${ }^{38}$ ONUSIDA (2002). «Prise en charge, traitement et soutien des personnes vivant avec le VIH/SIDA, Rapport sur l'épidémie mondiale de VIH/SIDA 2002 », Page 147157. Genève : ONUSIDA.

${ }^{39}$ UNAIDS (2009) «What Countries Need: Investments Needed for 2010 Targets ». UNAIDS February 2009. 24p.

${ }^{40}$ Carrin, G. et James, C. (2006): «L'assurance maladie obligatoire : Transition vers la couverture universelle et évaluation de la performance », chapitre 16 dans G. Dussault, P. Fournier, A. Letourmy (eds) «L'Assurance maladie en Afrique francophone », Banque Mondiale. 
${ }^{41}$ Lange, J.M., Schellekens, O.P., Lindner, M. and Van Der Gaag, J., (2008). "Public-Private Partnerships and New Models of Healthcare Access." Current Opinion in HIV and AIDS, Volume 3, July 2008, p.509-513.

${ }^{42}$ Huybrechts, A. and Fontenau, B. (2005). Microfinance institutions \& HIV/AIDS Transversal Analysis of Existing Initiatives. Flemish Interuniversity Council (VLIR) \& Belgian Development Cooperation (DGDC). Belgique: Université Catholique de Louvain.

${ }^{43}$ Chakonta, A. (2007). "A Case Study of Madison Insurance Company Zambia Ltd. Microinsurance \& HIV/AIDS in Zambia. Expert Group Meeting Innovative Finance for Sustainable Development.". Présented at the Microinsurance Conference, 18-20 October 2005. Munich.

${ }^{44}$ Parker, J., Singh, I. and Hattel, K. (2000). UNAIDS Background Paper, The Role of Microfinance in the Fight Against HIV/AIDS, A Report to the Joint United Nations Program on HIV/AIDS (UNAIDS). Bethesda: Development Alternatives, Inc.

${ }^{45}$ Develtere, P., Doyen, G. and Fonteneau, B. (2004). Micro-insurance and Health Care in Developing Countries An International Picture, Cera Foundation: Leuven. [Consulté le 16/04/2010] URL: http:// www.cooperatiefondernemen.be/publicaties/R941c.pdf.

\section{NOTES}

${ }^{a}$ La lutte contre le VIH/SIDA rentre dans le cadre du 6ème objectif des OMD : Combattre le VIH/SIDA, le paludisme et les autres maladies. Il est question dans les cibles 1 et 2 de ce $6^{\text {e }}$ objectif d'enrayer, voire de faire reculer la pandémie du VIH et du SIDA d'ici 2015, et favoriser un accès universel des traitements disponibles contre le VIH et le Sida en 2010.

bSans oublier l'aide financière considérable apportée par des organisations à but non lucratif comme Bill and Melinda Gate Foundation, Clinton Foundation.

${ }^{c}$ Le plan d'aide d'urgence du Président Bush en matière de lutte contre le SIDA

${ }^{\mathrm{d}}$ UNAIDS, Resource needs for an expanded response to AIDS in low and middle income countries. Disponible sur: www.unaids.org/html/pub/publications/ircpub06/resource needsreport_24jun05_en_pdf.pdf, consulté le 19 septembre 2010.

eAssemblée générale des Nations Unies, consacrant le consensus international en faveur d'une approche globale conjuguant prévention et soins, y compris accès aux ARV. 